\title{
Influencia de la virtualidad en la deserción de estudiantes en la Universidad Nacional Abierta y a Distancia. UNAD - CEAD, Yopal (Casanare - Colombia')
}

Deyanira Mendoza García

Martín Gómez Orduz ${ }^{3}$

\begin{abstract}
Resumen
Este artículo presenta los resultados de la investigación realizada sobre el impacto que ha tenido la mediación virtual en la educación abierta y a distancia, y si ésta influye en la deserción de los estudiantes en la UNAD CEAD Yopal, para que posteriormente se formulen los correctivos pertinentes que permitan fortalecer y fomentar la educación a distancia.

El tipo de metodología empleado en esta investigación es mixto, ya que se empleó el método cuantitativo y el cualitativo, por lo que la aplicación de este método implicó el diseño de una encuesta con la cual se pretendía identificar las causas de la deserción de los estudiantes en la UNAD CEAD Yopal.
\end{abstract}

1 Esta investigación se realizó como tesis para la Maestría Higher Education (UNAD FL)

2 Universidad Nacional Abierta y a Distancia, tutor ECEDU y Decana Espejo de la ECEDU en la ZAO, licenciada en Lenguas Modernas, especialista en Evaluación Educativa Institucional, M.A. en Education - Higer Education (UNAD FL), deyanira. mendoza@unad.edu.co Colombia

3 Universidad Nacional Abierta y a Distancia, tutor ECBTI y Líder Zonal de Investigación ZAO, licenciado en Física y Matemáticas (UPTC), especialista en Educación Superior a Distancia (UNAD), M.A. Specialization in Online Education (UNAD FL), martin.gomez@unad.edu.co Colombia. 
Palabras clave: educación a distancia, estudiante, medio y mediaciones pedagógicas, deserción, TIC, campus virtual, Aprendizaje Autónomo, Clases de Evaluación.

\title{
Influence of virtual system in school dropping out at the Universidad Nacional Abierta y a Distancia. UNAD - CEAD, Yopal (Casanare - Colombia)
}

\begin{abstract}
This paper presents the findings of a research study on the impact of virtual mediation in open and distance higher educuation, and how much influence it has in student desertion at UNAD CEAD Yopal, so that pertinent correctives may be formulated in order to strengthen and foster distance education. The methodology followed for this research was mixed, as methods used were quantitative and qualitative, through a survey designed to identify the causes of desertion among students at UNAD - CEAD, Yopal.
\end{abstract}

Key words: Distance education, student, pedagogical mediations and means, desertion, ICT's, virtual campus, autonomous learning, types of evaluation.

\section{Introducción}

En este artículo se presentan los resultados de la investigación «Influencia de la virtualidad en la deserción de estudiantes en la Universidad Nacional Abierta y a Distancia. UNAD CEAD YOPAL Casanare - Colombia», por medio de la cual se pretendía conocer la aceptabilidad que ha tenido el proceso 
educativo a través de la mediación virtual, siendo esta, la que ofrece la posibilidad de brindar una educación para todos a través de la modalidad abierta y a distancia, con libertad académica como pilar integrador y responsabilidad sustantiva del aprendizaje autónomo, centrado en la auto gestión formativa mediante el uso pedagógico apropiado e intensivo de las tecnologías de la información y la comunicación que garantice la excelencia académica y la democratización como compromiso con el acceso y la sostenibilidad de la información en la gestión organizacional y global del conocimiento.

Partiendo de la premisa de que la educación a distancia cada día se va fortaleciendo, en la medida que facilita a los gobiernos territoriales ampliar las coberturas a menor costo, al no tener que invertir en infraestructura física para dar cumplimiento a la obligación constitucional que le asiste al Estado de propiciar los medios para garantizar la educación de sus comunidades.

Para el desarrollo de la investigación fue necesario diseñar una encuesta con la que se recogió la información requerida para este estudio. El elemento central que se tuvo en cuenta fue el hecho de que se espera que la matricula en mediación virtual aumente significativamente, para lo cual es necesario buscar los factores y variables de la resistencia al uso del campus virtual con el fin de generar las estrategias necesarias que motiven y faciliten la utilización de esta mediación pedagógica.

\section{Metodología}

El tipo de metodología que se empleó en esta investigación fue de carácter mixto, en el sentido de que hay elementos del método cuantitativo y del método cualitativo. Ahora bien, sobre el primero de estos Cook y Reichart (citados por Meza) dicen: «Cuando se aplican métodos cuantitativos se miden características o variables que pueden tomar valores numéricos y deben describirse para facilitar la búsqueda de posibles relaciones mediante el análisis estadístico. En este caso el instrumento a aplicar fue la encuesta con la cual se identificaron las causas de la deserción del estudiante en la UNAD CEAD Yopal. Por otra parte, en cuanto al método cualitativo, Rodríguez Gómez (1996), señala que es un método de investigación usado principalmente en las ciencias sociales que se basa en cortes metodológicos basados en principios teóricos tales como la fenomenología, la hermenéutica y la interacción social. Debido a que se revisarán algunas de las estrategias que emplean los consejeros y tutores para evitar la deserción de los estudiantes de los cursos del campus virtual, la investigación se desarrolló en las siguientes etapas: 
- Formulación de la propuesta de investigación.

- Documentación.

- Recolección de la información entre el personal académico por medio de una encuesta.

- Análisis y tabulación de la encuesta.

- Presentación del informe final.

\section{Población}

Los participantes fueron estudiantes que desertaron de sus programas de estudio y que poseen distintas características sociales económicas y culturales. Fue necesario la utilización de la base de datos de registro y control de la universidad para ubicarlos, lo que permitió la aplicación de la encuesta a 88 estudiantes, que representan la muestra tomada de la población estudiantil desertada del 2006 al 2009, en la Unad Cead Yopal.

\section{Instrumentos}

Uno de los instrumentos empleados para la recolección de información fue el análisis del Plan Alerte, elaborado por el Sistema de Soporte y Consejería Virtual, que consiste en un aplicativo que recolecta la siguiente información: nombre y código del curso académico, nombre del tutor y director del curso, número de estudiantes matriculados, información personal del estudiante, la cual permite ubicarlo en un programa académico y semestre, número de estudiantes que no han ingresado al aula, los que registran más de diez días de ausencia y los que ingresan pero no tienen registro de actividades.

Para la recolección de información primaria se diseñó una encuesta con un total de 15 preguntas con la que se identificó, si la mediación virtual fue el factor de deserción en la UNAD CEAD Yopal.

\section{Método de análisis de datos}

Se utilizaron métodos estadísticos para analizar los datos recolectados, así como programas como Excel para el manejo de los datos y obtener resultados estadísticos, que permitan, además de sus análisis, la representación de los datos en figuras.

\section{Resultados del análisis de datos}

El estudio de campo fue aplicado a una población de 953 estudiantes cuya muestra calculada, con la fórmula estadística para poblaciones finitas, es de 
88 estudiantes de la UNAD CEAD Yopal, para identificar los factores de deserción, lo que arrojó los siguientes resultados:

\begin{tabular}{|l|l|l|}
\hline $\mathbf{X i}$ & $\mathbf{F i}$ & Porcentaje (\%) \\
\hline Urbano & 36 & $41 \%$ \\
\hline Rural & 52 & $59 \%$ \\
\hline Total & 88 & $100 \%$ \\
\hline
\end{tabular}

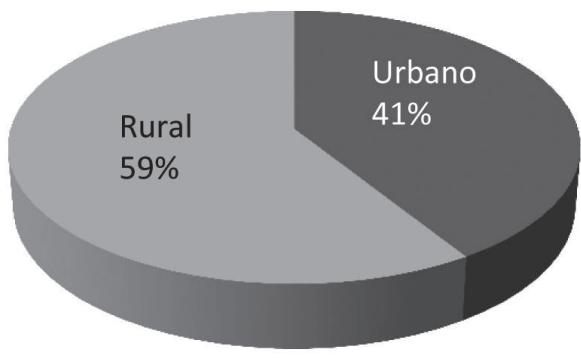

Figura 1. Lugar de residencia de los estudiantes desertores

Fuente: Los autores

E1 59\% de los estudiantes que desertan viven en el área rural, lo que dificulta el acceso a los medios tecnológicos por no tener conectividad; el $41 \%$ residen en el área urbana.

Tabla 1. Edades de los estudiantes desertores

\begin{tabular}{|l|l|l|l|l|l|l|l|l|}
\hline \multicolumn{2}{|l|}{$\mathbf{X i - 1}-\mathbf{X i}$} & $\mathbf{f i}$ & $\mathbf{h i}$ & $\mathbf{F i}$ & $\mathbf{H i}$ & $\mathbf{f} \%$ & $\mathbf{X i}$ & $\mathbf{X}{ }^{\star} \mathbf{f i}$ \\
\hline 15,1 & 25 & 43 & 0,49 & 43 & 0,49 & 49 & 20 & 860 \\
\hline 25,1 & 35 & 19 & 0,22 & 62 & 0,70 & 22 & 30 & 570 \\
\hline 35,1 & 45 & 12 & 0,14 & 74 & 0,84 & 14 & 40 & 480 \\
\hline 45,1 & 55 & 9 & 0,10 & 83 & 0,94 & 10 & 50 & 450 \\
\hline 55,1 & 65 & 5 & 0,06 & 88 & 1 & 6 & 60 & 300 \\
\hline Total & & 88 & 1 & & & 100,0 & & 2660 \\
\hline
\end{tabular}

Media $\mu=30,2$ Años; Mediana $M e=25,3$ Años; Moda $M d=22,0$ Años

Fuente: Los autores. 


\section{Deyanira Mendoza García y Martín Gómez Orduz}

Influencia de la virtualidad en la deserción de estudiantes en la Universidad Nacional Abierta y a Distancia. UNAD - CEAD,

Yopal (Casanare - Colombia), artículo producto de la investigación

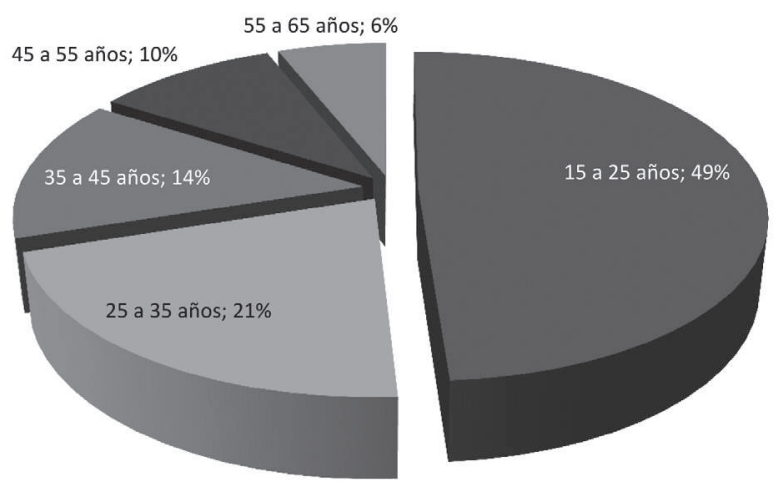

Figura 2. Edades de los estudiantes desertores

Fuente: Los autores

El $49 \%$ de los estudiantes desertores se encuentra en una edad entre los 15 y los 25 años, debido a que son estudiantes que vienen de una modalidad de educación presencial y no tienen manejo de tiempo y disciplina para un proceso de autoformación. El 21\% pertenece a los estudiantes entre 25 y 35 años, generalmente se da por dificultades económicas, este segmento de población aborda el proceso con más seriedad; el $14 \%$ entre 35 y 45 años, se da por manejo de herramientas informáticas, igual sucede con el 10\% entre 45 y 55 años y el $6 \%$ de los 55 a los 65 años.

\begin{tabular}{|l|l|l|}
\hline $\mathbf{X i}$ & $\mathbf{F i}$ & Porcentaje (\%) \\
\hline Empleado & 35 & 40 \\
\hline Independiente & 18 & 20 \\
\hline Empresario & 15 & 17 \\
\hline Desempleado & 20 & 23 \\
\hline Total & 88 & 100 \\
\hline
\end{tabular}

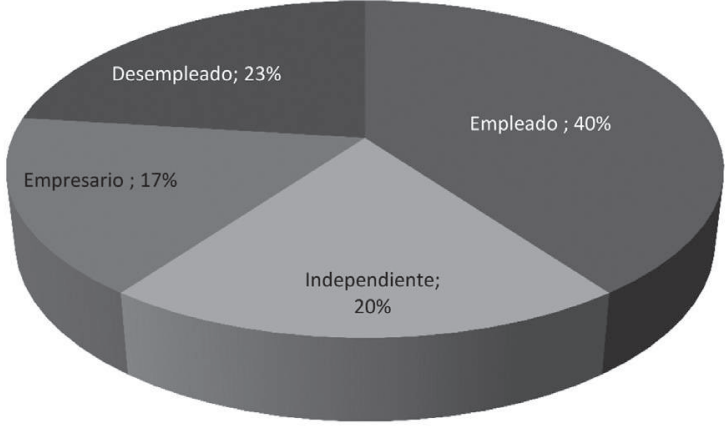

Figura 3. Actividad económica de los estudiantes

Fuente: Estudio de la investigación 
Las personas que desertan en un $40 \%$ son empleados, lo que les impide hacer una adecuada distribución de su tiempo y tener acceso a los medios tecnológicos, ya que, la mayor parte de la población estudiantil pertenece al estrato 1 y 2; le siguen la población desempleada con $23 \%$ que por falta de recursos económicos deben abandonar sus estudios; los trabajadores independientes desertan en un $20 \%$, debido a la competencia en la prestación de servicios; los empresarios son los que menos desertan porque tienen estabilidad económica y pueden disponer de su tiempo, además cuentan con los recursos tecnológicos requeridos para su proceso de aprendizaje.

\begin{tabular}{|l|l|l|}
\hline $\mathbf{X i}$ & $\mathbf{F i}$ & Porcentaje (\%) \\
\hline 1 y 3 integrantes & 20 & $23 \%$ \\
\hline 4 y 7 Integrantes & 65 & $74 \%$ \\
\hline $\begin{array}{l}\text { Más de 7 } \\
\text { Integrantes }\end{array}$ & 3 & $3 \%$ \\
\hline Total & 88 & $100 \%$ \\
\hline
\end{tabular}

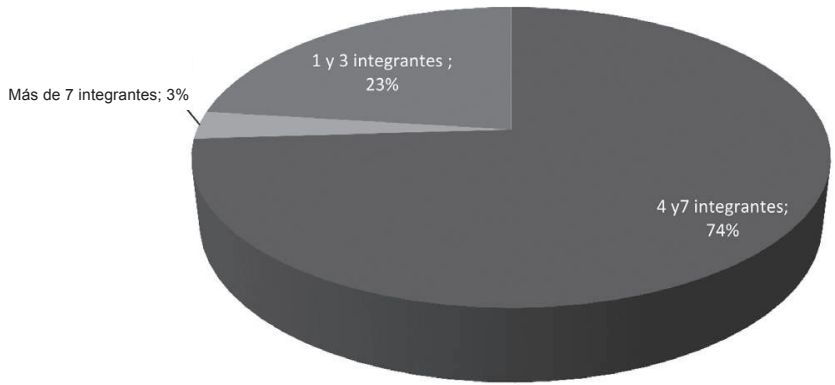

Figura 4. Conformación del núcleo familiar

El 74\% de los estudiantes desertores tienen un núcleo familiar conformado entre cuatro y siete personas, esto implica que se tengan dificultades económicas y se abandonen los estudios para cumplir con las necesidades básicas que requieren; el $23 \%$ conforman un grupo familiar entre 1 y tres personas; los estudiantes con más de siete integrantes en el núcleo familiar presentan el menor índice, debido a que son pocos los que tienen el acceso a la educación superior.

\begin{tabular}{|l|l|l|}
\hline $\mathrm{Xi}$ & $\mathrm{Fi}$ & Porcentaje (\%) \\
\hline $\begin{array}{l}\text { Menor o = a 1 } \\
\text { smmlv }\end{array}$ & 45 & $51 \%$ \\
\hline Entre 2 y 3 smmlv & 30 & $34 \%$ \\
\hline Más de 3 smmlv & 13 & $15 \%$ \\
\hline Total & 88 & $100 \%$ \\
\hline
\end{tabular}




\section{Deyanira Mendoza García y Martín Gómez Orduz}

Influencia de la virtualidad en la deserción de estudiantes en la Universidad Nacional Abierta y a Distancia. UNAD - CEAD,

Yopal (Casanare - Colombia), artículo producto de la investigación

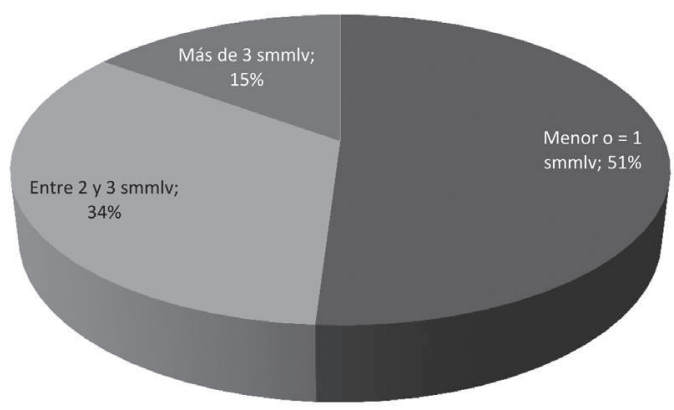

Figura 5. Nivel de ingresos de los estudiantes desertores

Fuente: Los autores

El $51 \%$ de los estudiantes que desertan devengan menos o igual a un salario mínimo mensual legal vigente, siendo el factor económico el principal causante de deserción; mientras que el 34\% devengan entre 2 y 3 salarios mínimos mensuales legales vigentes, el menor grado de deserción se presenta en quienes devengan más de tres salarios mínimos mensuales vigentes, quienes a pesar de tener más solidez económica por sus múltiples ocupaciones y responsabilidades se les dificulta continuar con su proceso de aprendizaje.

\begin{tabular}{|l|l|l|}
\hline $\mathbf{X i}$ & $\mathbf{F i}$ & Porcentaje (\%) \\
\hline Recursos Propios & 35 & $40 \%$ \\
\hline Crédito & 10 & $11 \%$ \\
\hline $\begin{array}{l}\text { Convenio } \\
\text { Institucional }\end{array}$ & 10 & $11 \%$ \\
\hline Apoyo empleador & 13 & $15 \%$ \\
\hline Apoyo Familiar & 20 & $23 \%$ \\
\hline Total & 88 & $100 \%$ \\
\hline
\end{tabular}

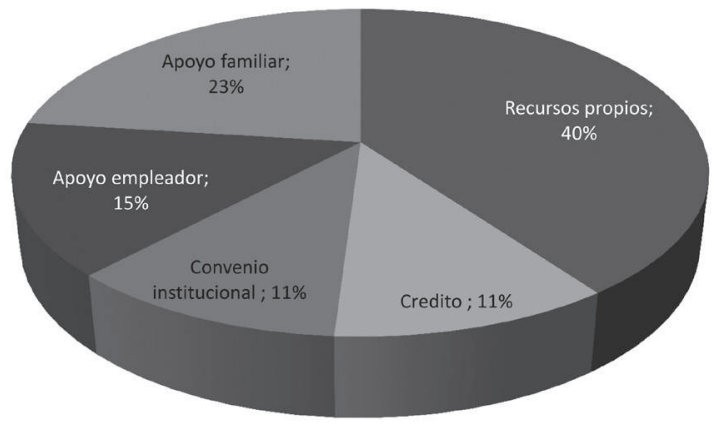

Figura 6. Financiación del estudio.

Fuente: Los autores 
El $40 \%$ de los desertores financian sus estudios con recursos propios, por ello dificulta su continuidad al no tener los recursos disponibles para sufragar los costos que demanda el proceso académico; el 23\% financian sus estudios con apoyo familiar, al no contar con éste apoyo por diferentes circunstancias se ven en la obligación de abandonar sus estudios; el 15\% con apoyo del empleador, por la recepción económica y políticas de las empresas los contratos se dan a término fijo, al quedar desempleados pierden ese beneficio y deben retirarse al contar con el apoyo económico requerido; el 11\% se presenta en estudiantes que tienen convenio institucional y crédito, por lo general se da en estudiantes que no tienen un sitio de trabajo permanente, deben estar viajando por el país para cumplir con sus labores para las cuales fueron contratados, lo que dificulta el proceso.

\begin{tabular}{|l|l|l|}
\hline $\mathbf{X i}$ & $\mathbf{F i}$ & Porcentaje (\%) \\
\hline Café internet & 10 & $11 \%$ \\
\hline $\begin{array}{l}\text { Plan residencial o } \\
\text { modem }\end{array}$ & 15 & $17 \%$ \\
\hline $\begin{array}{l}\text { Servicio en la oficina } \\
\text { donde labora }\end{array}$ & 25 & $29 \%$ \\
\hline Sede universitaria & 38 & $43 \%$ \\
\hline Total & 88 & $100 \%$ \\
\hline
\end{tabular}

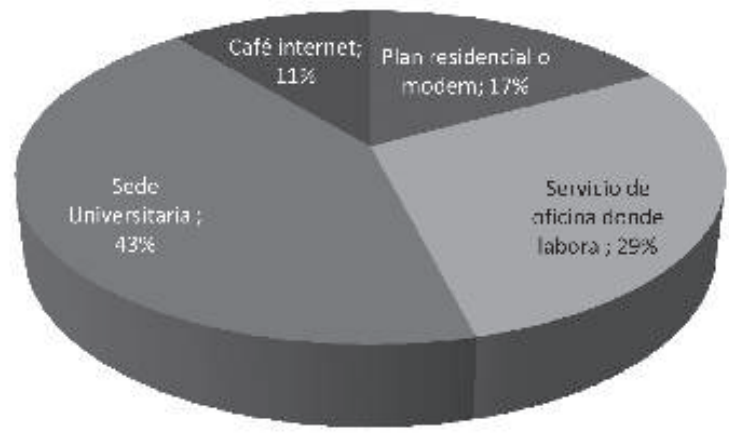

Figura 7. Lugar de acceso a Internet.

Fuente: Los autores

Una de las dificultades por la que desertan los estudiantes son: El acceso al servicio de Internet, el $43 \%$ de la población manifiesta tener acceso al servicio en el Cead (Centro de educación a distancia), siendo éste su único lugar de acceso, cuyo servicio se limita a un horario de ingreso; el 29\% acceden en el lugar de trabajo, esto entorpece sus funciones y no permite mayor grado de concentración; el 17\% manifiesta tener plan residencial, facilitando su proceso de aprendizaje; el $11 \%$ hace uso de cafés Internet incrementado sus costos educativos. 


\section{Deyanira Mendoza García y Martín Gómez Orduz}

Influencia de la virtualidad en la deserción de estudiantes en la Universidad Nacional Abierta y a Distancia. UNAD - CEAD, Yopal (Casanare - Colombia), artículo producto de la investigación

\begin{tabular}{|l|l|l|}
\hline $\mathbf{X i}$ & $\mathbf{F i}$ & Porcentaje (\%) \\
\hline SI & 35 & $40 \%$ \\
\hline NO & 53 & $60 \%$ \\
\hline Total & 88 & $100 \%$ \\
\hline
\end{tabular}

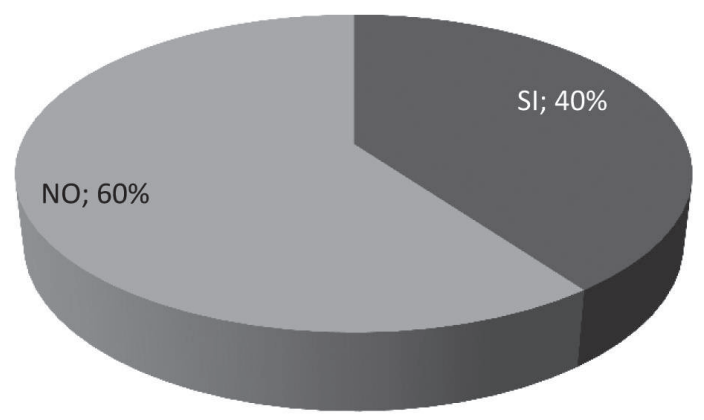

Figura 8. Conocimiento del aprendizaje autónomo antes de ingresar a la UNAD.

Fuente: Los autores

El $60 \%$ de los estudiantes desertores manifiestan no haber conocido antes de ingresar a la UNAD Cead Yopal la modalidad de educación a distancia, ésta exige autodisciplina y manejo de tiempo, por ello quienes abandonan sus estudios en mayor porcentaje es la población entre 15 y 25 años.

\begin{tabular}{|l|l|l|}
\hline $\mathbf{X i}$ & $\mathbf{F i}$ & Porcentaje (\%) \\
\hline Ninguno & 42 & $48 \%$ \\
\hline Regular & 24 & $27 \%$ \\
\hline Bueno & 22 & $25 \%$ \\
\hline Total & 88 & $100 \%$ \\
\hline
\end{tabular}

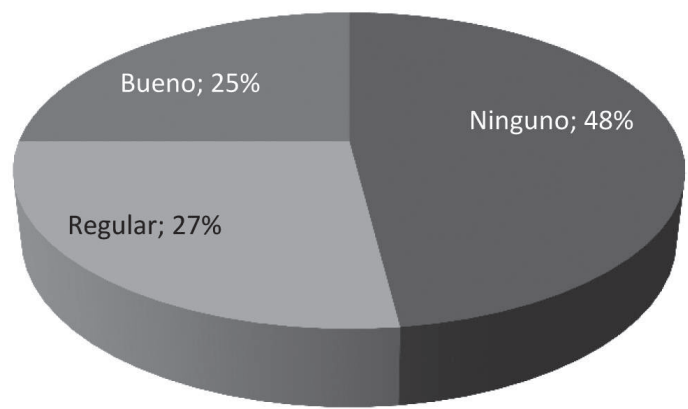

Figura 9. Manejo de herramientas informáticas

Fuente: Los autores 


\section{Revista de}

El 48\% de los estudiantes desertores al ingresar a la universidad no tenía ningún conocimiento sobre el manejo de herramienta informáticas, situación que dificultó el proceso de aprendizaje en ambientes virtuales; El $27 \%$ manejaban regularmente el sistema, y sólo el $22 \%$ tenía un buen conocimiento de las herramientas que le facilitaban el proceso de aprendizaje autónomo en ambientes virtuales, sin embargo por el acceso al servicio de conectividad se vieron obligados a abandonar sus estudios.

\begin{tabular}{|l|l|l|}
\hline $\mathbf{X i}$ & $\mathbf{F i}$ & Porcentaje (\%) \\
\hline SI & 26 & $30 \%$ \\
\hline NO & 62 & $70 \%$ \\
\hline Total & 88 & $100 \%$ \\
\hline
\end{tabular}

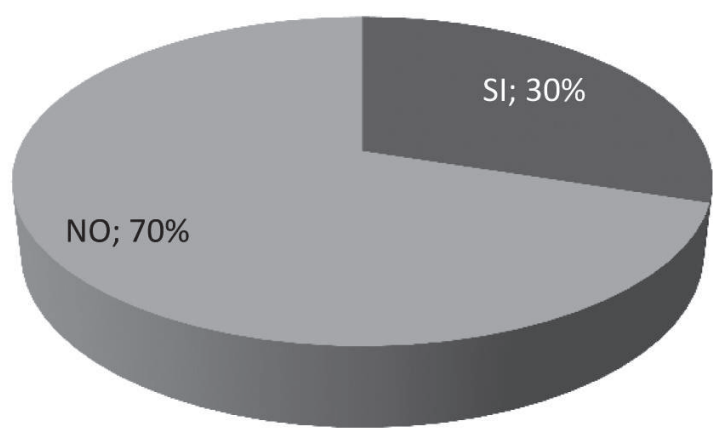

Figura 10. Facilidad del uso de las TIC en el proceso de aprendizaje autónomo.

Fuente: Los autores

\begin{tabular}{|l|l|l|}
\hline $\mathbf{X i}$ & $\mathbf{F i}$ & Porcentaje (\%) \\
\hline $\begin{array}{l}\text { No posee } \\
\text { Computador }\end{array}$ & 15 & $17 \%$ \\
\hline $\begin{array}{l}\text { No maneja el Campus } \\
\text { Virtual }\end{array}$ & 25 & $29 \%$ \\
\hline $\begin{array}{l}\text { Dificultad en trabajo } \\
\text { colaborativo }\end{array}$ & 25 & $28 \%$ \\
\hline $\begin{array}{l}\text { Incremento del costo de } \\
\text { estudio por el uso de } \\
\text { Internet }\end{array}$ & 23 & $26 \%$ \\
\hline Total & 88 & $100 \%$ \\
\hline
\end{tabular}




\section{Deyanira Mendoza García y Martín Gómez Orduz}

Influencia de la virtualidad en la deserción de estudiantes en la Universidad Nacional Abierta y a Distancia. UNAD - CEAD,

Yopal (Casanare - Colombia), artículo producto de la investigación

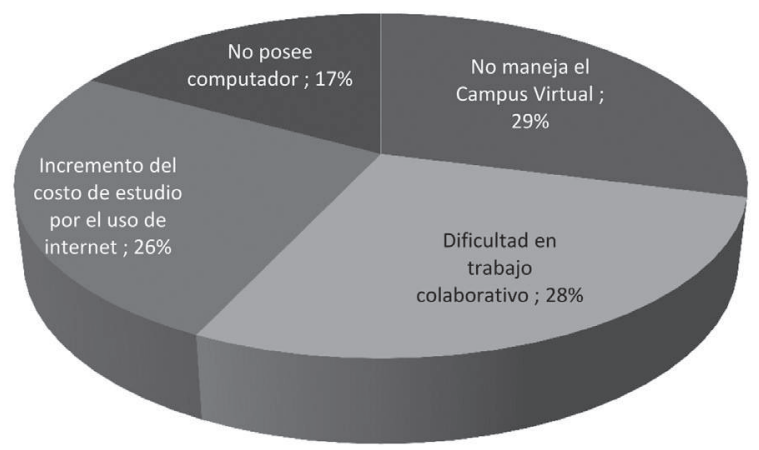

Figura 11. Dificultades para el uso de la TIC.

Fuente: Los autores

El 70\% de la población desertora manifestó tener dificultades en el uso de las TIC como herramientas para el proceso de aprendizaje autónomo porque no maneja el campus virtual en un $29 \%$, presenta incompatibilidad para el desarrollo de trabajos colaborativos en un $28 \%$. El $30 \%$ se le facilita el aprendizaje a través de éste medio, dentro de ellos un $26 \%$ manifiesta que esta modalidad incrementa los costos ya que su acceso al internet es limitado; además al $17 \%$ no posee computador.

\begin{tabular}{|l|l|l|}
\hline $\mathbf{X i}$ & $\mathbf{F i}$ & Porcentaje (\%) \\
\hline Algunas veces & 48 & $54 \%$ \\
\hline Frecuentemente & 28 & $32 \%$ \\
\hline Nunca & 12 & $14 \%$ \\
\hline Total & 88 & $100 \%$ \\
\hline
\end{tabular}

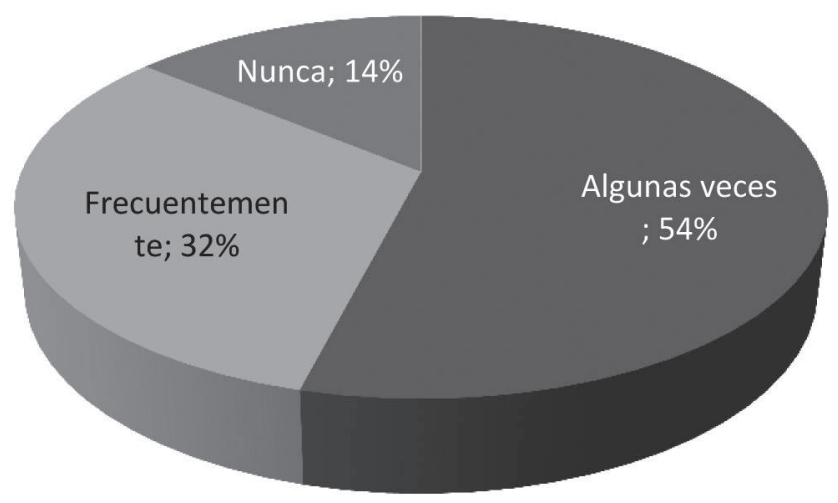

Figura 12. Frecuencia de participación en trabajos colaborativos

Fuente: Los autores. 
El trabajo colaborativo es una actividad que permite la interacción entre estudiantes y tutores al igual que la puesta en práctica de los conceptos en cada texto; sin embargo se evidencia una alta resistencia al mismo representada en el 54\% de los estudiantes desertores; el 32\% participaba frecuentemente pero tenía dificultades al acceso de conectividad y el 14\% nunca participó por no tener conocimiento del manejo de las herramientas didácticas del campus.

\begin{tabular}{|l|l|l|}
\hline $\mathbf{X i}$ & $\mathbf{F i}$ & Porcentaje (\%) \\
\hline $\begin{array}{l}\text { Dentro de las 24 } \\
\text { horas }\end{array}$ & 13 & $15 \%$ \\
\hline De 24 a 48 horas & 23 & $26 \%$ \\
\hline $\begin{array}{l}\text { Después de 48 } \\
\text { horas }\end{array}$ & 34 & $39 \%$ \\
\hline $\begin{array}{l}\text { No recibió res- } \\
\text { puesta }\end{array}$ & 18 & $20 \%$ \\
\hline Total & 88 & $100 \%$ \\
\hline
\end{tabular}

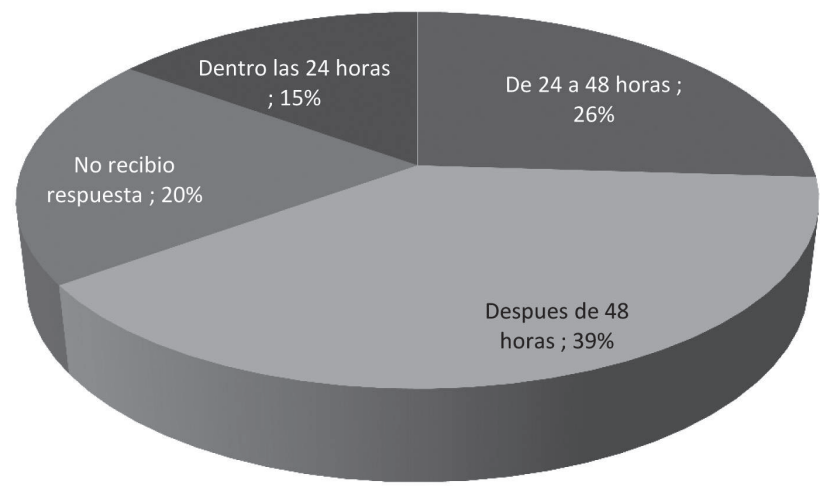

Figura 13. Tiempo de respuesta por parte del tutor Fuente: Los autores.

El 39\% de los desertados manifestaron recibir respuesta después de 48 horas, debido al alto número de estudiantes por tutor, se dificulta dar respuesta dentro de las 24 horas, estando éste tiempo representado en un 15\%; El 26\% afirman haber recibido retroalimentación a sus inquietudes en un tiempo entre 24 y 48 horas y un $20 \%$ manifiesta no haber recibido respuesta. El tiempo de respuesta en un proceso de aprendizaje en ambientes virtuales desmotiva al estudiante.

\begin{tabular}{|l|l|l|}
\hline $\mathbf{X i}$ & $\mathbf{F i}$ & Porcentaje (\%) \\
\hline $\mathrm{SI}$ & 39 & $30 \%$ \\
\hline
\end{tabular}




\section{Deyanira Mendoza García y Martín Gómez Orduz}

Influencia de la virtualidad en la deserción de estudiantes en la Universidad Nacional Abierta y a Distancia. UNAD - CEAD, Yopal (Casanare - Colombia), artículo producto de la investigación

\begin{tabular}{|l|l|l|}
\hline $\mathbf{X i}$ & $\mathbf{F i}$ & Porcentaje (\%) \\
\hline NO & 49 & $70 \%$ \\
\hline Total & 88 & $100 \%$ \\
\hline
\end{tabular}

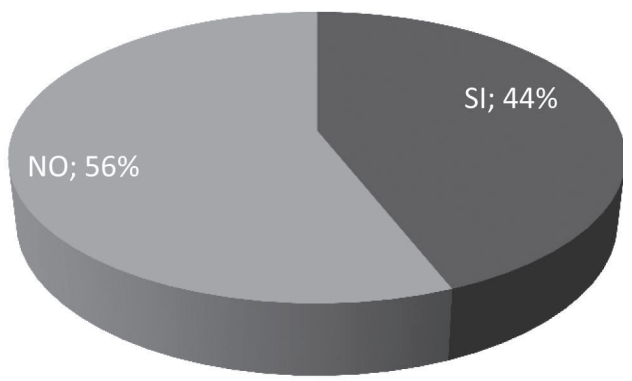

Figura 14. Pertinencia del material didáctico Fuente: Los autores

El $56 \%$ de los desertores no consideran pertinente el material didáctico dispuesto para un proceso de aprendizaje autónomo a través de los ambientes virtuales, por su estructura plana, se dificulta su comprensión, a esto se suma la poca disponibilidad de los tutores por el alto número de estudiantes que manejan, impidiendo que se dé una interacción más activa que facilite la comprensión del mismo.

\begin{tabular}{|l|l|l|}
\hline $\mathbf{X i}$ & $\mathbf{F i}$ & Porcentaje (\%) \\
\hline $1-2$ & 0 & $0 \%$ \\
\hline $2.1-3$ & 15 & $17 \%$ \\
\hline $3.1-4$ & 42 & $48 \%$ \\
\hline $4.1-4.9$ & 31 & $35 \%$ \\
\hline 5 & 0 & $0 \%$ \\
\hline Total & 88 & $100 \%$ \\
\hline
\end{tabular}

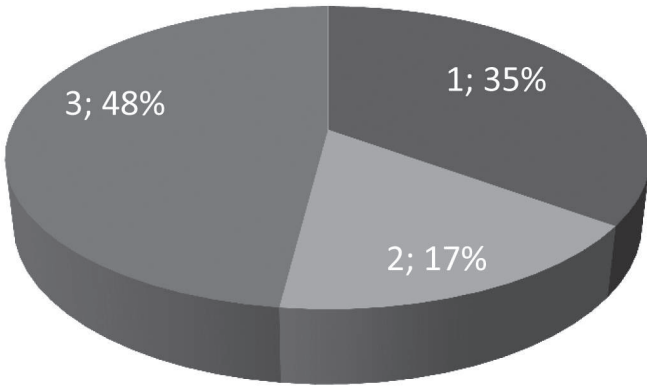

Figura 15. Grado de aprendizaje con el uso de las TIC de 1 a 5.

Fuente: Los autores 


\section{Revista de \\ investigaciones UNAD \\ Volumen 11. Número 1. Junio 2012}

El 48\% de los desertados califica el aprendizaje en ambientes virtuales con nota de 3.1 y 4.0 ; el $35 \%$ lo califica entre 4.1 y 4.9 ; el $17 \%$ lo evalúa entre 2.1 y 3.0 .

\begin{tabular}{|l|l|l|}
\hline $\mathbf{X i}$ & $\mathbf{F i}$ & Porcentaje (\%) \\
\hline SI & 65 & $74 \%$ \\
\hline NO & 23 & $26 \%$ \\
\hline Total & 88 & $100 \%$ \\
\hline
\end{tabular}

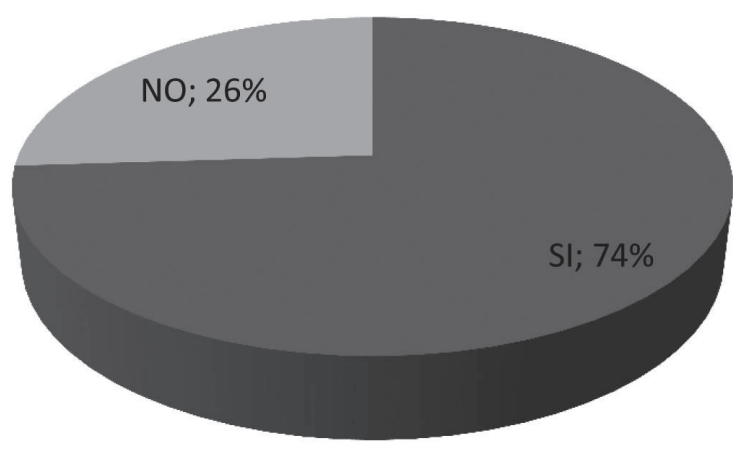

Figura 16. Uso de mediación virtual en el proceso de aprendizaje Fuente: Los autores

\begin{tabular}{|l|l|l|}
\hline $\mathbf{X i}$ & $\mathbf{F i}$ & Porcentaje (\%) \\
\hline $\begin{array}{l}\text { Menos de una } \\
\text { hora }\end{array}$ & 19 & $22 \%$ \\
\hline Entre 1 y 2 horas & 22 & $25 \%$ \\
\hline Más de 2 horas & 47 & $53 \%$ \\
\hline Total & 88 & $100 \%$ \\
\hline
\end{tabular}

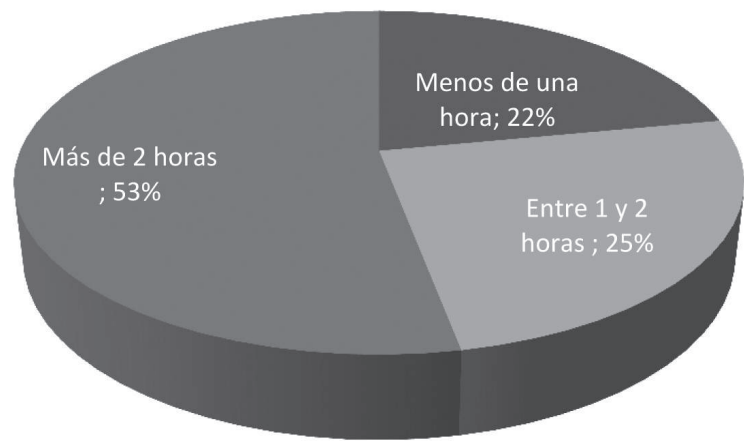

Figura 17. Tiempo diario dedicado al proceso de estudio

Fuente: Los autores 


\section{Deyanira Mendoza García y Martín Gómez Orduz}

Influencia de la virtualidad en la deserción de estudiantes en la Universidad Nacional Abierta y a Distancia. UNAD - CEAD,

Yopal (Casanare - Colombia), artículo producto de la investigación

El 74\% de los estudiantes desertados hicieron uso del proceso de aprendizaje a través de la mediación virtual, el tiempo que dedicaron diario fue más de dos horas, un $25 \%$ dedicó entre una y dos horas y un $19 \%$ menos de una hora; el $26 \%$ no hizo uso de los ambientes virtuales de aprendizaje, lo que significa que eran estudiantes con mediación tradicional.

\begin{tabular}{|l|l|l|}
\hline $\mathbf{X i}$ & $\mathbf{F i}$ & Porcentaje (\%) \\
\hline $\begin{array}{l}\text { Aprendizaje a } \\
\text { través de las TIC }\end{array}$ & 42 & $48 \%$ \\
\hline $\begin{array}{l}\text { Supresión de la } \\
\text { mediación } \\
\text { tradicional }\end{array}$ & 25 & $28 \%$ \\
\hline $\begin{array}{l}\text { Dificultades } \\
\text { económicas }\end{array}$ & 15 & $17 \%$ \\
\hline $\begin{array}{l}\text { Dificultades de } \\
\text { aprendizaje } \\
\text { autónomo }\end{array}$ & 6 & $7 \%$ \\
\hline Total & 88 & $100 \%$ \\
\hline
\end{tabular}

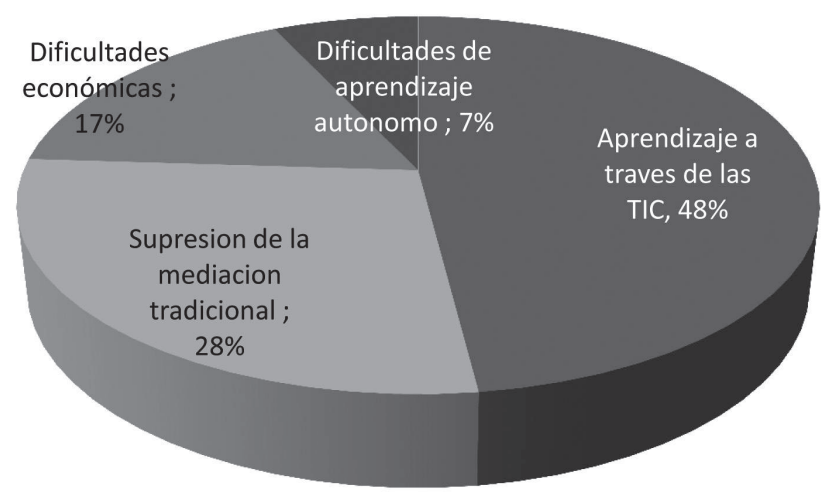

Figura 18. Motivo de deserción

Fuente: Los autores.

El 48\% de los estudiantes que desertaron utilizaban la mediación virtual en su proceso de aprendizaje, cuya dificultad en el manejo de las herramientas, la plataforma, el trabajo colaborativo, la tardanza en la respuesta de los tutores y el inadecuado material didáctico los llevaron a tomar ésta decisión. El 28\% manifiestan que fue la supresión de la mediación tradicional, el 17\% por dificultades económicas y un porcentaje mínimo representado en el 7\% por la modalidad de aprendizaje autónomo. 


\section{Discusión, conclusiones y recomendaciones}

Teniendo en cuenta el análisis de la información de campo y la información secundaria que sustenta esta investigación se puede concluir que en la Unad Cead Yopal la mediación virtual ejerce una gran influencia en la deserción de los estudiantes, cuyos factores son:

- Ubicación del lugar de residencia fuera del casco urbano de los municipios, lo que dificulta el uso de la mediación virtual debido a la baja conectividad en esos lugares.

- A la población joven se le dificulta el proceso de aprendizaje autónomo por la responsabilidad que requiere y el cambio de modelo pedagógico presencial al proceso de formación a distancia.

- La población estudiantil desertora de la Unad es una población trabajadora dependiente; la causa se fundamenta en la dificultad para distribuir el tiempo entre el trabajo y las horas de estudio.

- Otro grupo de desertores son los estudiantes que tienen un núcleo familiar superior a cuatro integrantes, y tienen dificultades económicas para suplir sus necesidades básicas, sobre todo aquellos que devengan menos de dos salarios mínimos. Este grupo de estudiantes en un alto porcentaje financian sus estudios con recursos propios, a pesar de existir facilidades con créditos como Aces e Icetex, debido a que la consecución de un codeudor presenta una gran dificultad para ellos.

- El acceso a internet es limitado, este sube los costos educativos, teniendo en cuenta que la población estudiantil es en general trabajadora con pocos ingresos, en general de estratos uno y dos, y que debido a su situación económica no pueden contar con una conexión a la red en sus hogares y el servicio dispuesto en el Cead en horas nocturnas es limitado.

- Existe un analfabetismo virtual lo que dificulta la apropiación de las herramientas, aunado al alto costo del servicio de internet, como ya se ha indicado.

- La mediación tradicional es una alternativa pero debido a los parámetros colocados por la universidad para brindar ésta modalidad con un mínimo de 20 estudiantes se está quitando la posibilidad a aquellos que no poseen los medios tecnológicos para abordar su proceso y se ven en la obligación de retirarse. 
- Debido al desconocimiento de las herramientas del campus virtual, La asignación de roles en los trabajos colaborativos y la falta de compromiso de los estudiantes en la realización de los mismos, no ejecutan estas actividades, generando desconcierto y desmotivación por la calificación asignada y la dificultad para superar el porcentaje perdido.

- El alto número de estudiantes asignado al tutor, impide que éste de respuesta a tiempo a todas las inquietudes de los estudiantes, y brinde un adecuado acompañamiento al proceso.

\section{Conclusiones}

A la sociedad actual hoy se suman las nuevas tecnologías de información esta ha cambiado fundamentalmente el proceso de educación de las personas, el conocimiento ya no está reservado para el tutor ni en las instituciones de educación, en la actualidad cada persona debe desempeñar un rol activo en la adquisición del conocimiento, hoy más que nunca el término autodidacta, auto-aprendizaje ha cobrado validez y los educadores tienen que enfrentar a una comunidad más exigente y autónoma. Estas nuevas tecnologías, facilitan y ofrecen nuevas alternativas educativas, como la educación virtual, la cual centra su atención en la producción del conocimiento versátil, rápido y oportuno basado en el principio de la creación de la comunidad educativa e interactiva y social del docente, estudiante e institución; fundamentado el modelo pedagógico en el aprendizaje activo, colaborativo y participativo, en donde el estudiante es el constructor de su propio aprendizaje de forma abierta y flexible; el docente es el orientador y facilitador del proceso.

La vitalización educativa se enmarca en las tendencias globalizadoras del mercado y ante ellas debe tener capacidad de respuesta con fundamento además de pedagógico, social y cultural lo que hace necesario diversificar la oferta educativa con diferentes modelos y modalidades en relación al trabajo, a la preparación científica y humanística y a la vida social en todo sus aspectos.

De acurdo al trabajo de campo realizado y a la información secundaria analizada el mayor problema de deserción se da por analfabetismo en el manejo de ambientes virtuales de aprendizaje y la sobre carga de estudiantes asignados a tutores en el campus virtual, se requiere evaluar el tiempo necesario para hacer el seguimiento a cada estudiante en su proceso de aprendizaje. El material didáctico debe ser más interactivo para incentivar la utilización de la mediación virtual y una adecuada distribución del tiempo de los estudiantes para el proceso de aprendizaje autónomo. 


\section{Referencias bibliográficas}

Chute, Thompsom y Hancock 1999, How Nontraditional Bachelor of Science Degree. Technology Students Perceive Distance Learning.

Gilham, Bukner y Butt 1998 Causas de tipo académico y no académico de la deserción estudiantil en el primer módulo de la Licenciatura en Dirección de la Cultura Física y el Deporte Modalidad Virtual Presencial.

Hernández, Roberto, Carlos Fernández y Pilar Baptista 1988. Metodología de la investigación. México: McGraw-Hill.

Homer, Kempfer. 2004. "How to Reduce Dropouts in Distance Education". Documento de Trabajo. UNED. Costa Rica.

http://es.wikipedia.org/wiki/Deserci\%C3\%B3n Wikipedia la Enciclopedia libre (2010)

http://es.wikipedia.org/wiki/Educaci\%C3\%B3n_a_distanciaWikipedia la Enciclopedia libre (2010)

http://www.google.com.co/search?hl=es\&lr=\&rlz=1G1ACAW_ESCO349\&defl=es\& q=define:campus+virtual\&ei=iWVOS9_KOMeWtgeLxYHaDA\&sa=X\&oi=glossary_ definition \&ct=title\&ved=oCAcQkAE Definición de campus virtual.

http://www.inacap.cl/data/e_news/boletin19/boletin19.html

http://www.mineducacion.gov.co/1621/articles-85600_archivo_pdf3.pdf

http://www.proyectopv.org/1-verdad/serestudiante.htm La página de la Vida. (2000) Universidad Nacional Abierta y a Distancia. (2006) Reglamento académico.

Levene, Pedro. (s.f.). “La investigación de mercado”. En Monografías.com. (http:// www.monografias.com/trabajos22/investigacion-mercado/investigacion-mercado. shtml ) consultado en:

Mtra. Sonia Verónica Mortis Lozoya y otros, 2009. Depto. Educación del Instituto Tecnológico de Sonora Mtro.

Rodríguez Gómez, Gregorio, Javier Gil y Eduardo García. 1996. Metodología de la investigación cualitativa. Granada: Ediciones Aljibe.

Rodrígez Gómez 1996. Método cualitativo

Según Phipps y Merisotis 1999. La efectividad de la enseñanza virtual. 


\section{Deyanira Mendoza García y Martín Gómez Orduz}

Influencia de la virtualidad en la deserción de estudiantes en la Universidad Nacional Abierta y a Distancia. UNAD - CEAD,

Yopal (Casanare - Colombia), artículo producto de la investigación

Sistema de Soporte de Consejería. 2009. Informe sobre Deserción

UNAD - CEAD Yopal. 2008. Informe de inducción y reinducción.

Taylor, Steve J., y Robert Bogdan. 1980. Introducción a los métodos cualitativos de investigación. Buenos Aires: Paidós.

Universidad Nacional Abierta y a Distancia. 2008. PAP Solidario.

Universidad Nacional Abierta y a Distancia. 2006. Reglamento General Estudiantil.

Universidad Pedagógica Nacional (UPN). 2009. "La deserción estudiantil. Reto investigativo y estratégico asumido de forma integral por la UPN”. 